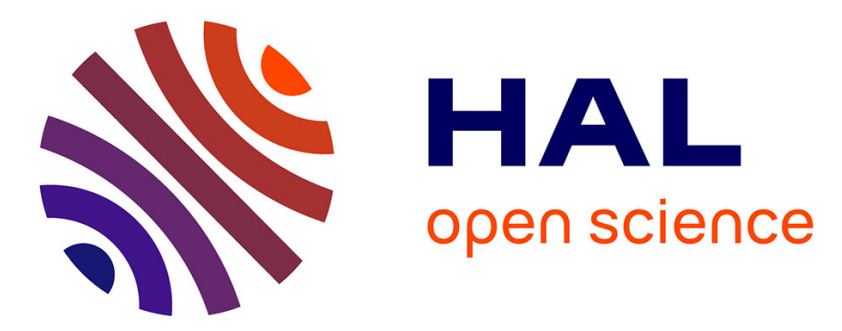

\title{
Eulerian versus Lagrangian simulation of unsteady two-way coupled coalescing two-phase flows in solid propellant combustion
}

François Doisneau, Joel Dupays, Angelo Murrone, Frédérique Laurent, Marc Massot

\section{To cite this version:}

François Doisneau, Joel Dupays, Angelo Murrone, Frédérique Laurent, Marc Massot. Eulerian versus Lagrangian simulation of unsteady two-way coupled coalescing two-phase flows in solid propellant combustion. Comptes Rendus Mécanique, 2013, 341, pp.44-54. 10.1016/j.crme.2012.10.005 . hal00875654

\section{HAL Id: hal-00875654 https://hal.science/hal-00875654}

Submitted on 22 Oct 2013

HAL is a multi-disciplinary open access archive for the deposit and dissemination of scientific research documents, whether they are published or not. The documents may come from teaching and research institutions in France or abroad, or from public or private research centers.
L'archive ouverte pluridisciplinaire HAL, est destinée au dépôt et à la diffusion de documents scientifiques de niveau recherche, publiés ou non, émanant des établissements d'enseignement et de recherche français ou étrangers, des laboratoires publics ou privés. 


\title{
Eulerian VS Lagrangian simulation of unsteady two-way coupled coalescing two-phase flows in solid propellant combustion
}

\author{
F. Doisneau ${ }^{\mathrm{a}, \mathrm{b}, \mathrm{c}}$, J. Dupays $^{\mathrm{a}}$, A. Murrone ${ }^{\mathrm{a}}$, F. Laurent $^{\mathrm{b}, \mathrm{c}}$ and M. Massot $^{\mathrm{b}, \mathrm{c}, \mathrm{d}}$ \\ ${ }^{a}$ ONERA - Département d'Énergétique Fondamentale et Appliquée (DEFA) - BP 81000, 91120 Palaiseau, FRANCE \\ ${ }^{\mathrm{b}}$ CNRS, UPR 288 - Laboratoire EM2C - Grande Voie des Vignes, 92295 Châtenay-Malabry, FRANCE \\ ${ }^{\mathrm{c}}$ Ecole Centrale Paris (ECP) - Grande Voie des Vignes, 92295 Châtenay-Malabry, FRANCE \\ ${ }^{\mathrm{d}}$ Center for Turbulence Research (CTR), Stanford University, California 94305-4035, USA
}

Received 15 November 2011; accepted after revision 30 June 2012

\begin{abstract}
Solid rocket motor flows depend strongly on alumina granulometry, which spreads up with coalescence. Solving droplet sizes in unsteady flows is a modeling and numerical challenge, specially when high loadings generate retrocoupling. As an alternative to Lagrangian approaches, the Eulerian Multi-Fluid model describes polydispersity with size-sorted droplet fluids called sections. A two size moment method, that spares sections, is implemented in the CEDRE code. A new transport and coupling method allows to capture moderately dense spray structures with low diffusion. The aim is threefold : compare CEDRE's Lagrangian and new Eulerian two-phase approaches, prove the feasibility of unsteady and complex motor flow simulations, and show the effect of coalescence on instabilities.

Résumé

Simulation eulérienne et lagrangienne d'écoulements diphasiques instationnaires avec coalescence et retrocouplage en propulsion solide. L'écoulement d'un moteur à propergol solide dépend fortement de la granulométrie d'alumine, qui s'étale avec la coalescence. Résoudre la taille des gouttes dans les écoulements instationnaires est un défi numérique et de modélisation, surtout quand les forts chargements génèrent du retrocouplage. Une alternative à l'approche lagrangienne est le modèle Multi-Fluide eulérien qui décrit la polydispersion en résolvant des fluides de gouttes triés par tailles, appelés sections. Une méthode à deux moments en taille, qui économise des sections, est implémentée dans le code CEDRE. Une nouvelle méthode pour le transport et le couplage permet de capturer les structures des sprays modérément denses avec une diffusion faible. L'objectif est triple : comparer les approches diphasiques lagrangienne et eulérienne améliorée de CEDRE, prouver la faisabilité de simulations d'écoulements complexes dans les moteurs et exhiber l'effet de la coalescence sur les instabilités.
\end{abstract}

Key words: Solid Rocket Motor ; Eulerian Multi-Fluid ; Coalescence ; Moderately dense spray

Mots-clés : Moteur à Propergol Solide; Multi-Fluide Eulérien; Coalescence; Spray modérément dense 


\section{Introduction}

In aluminized propellant combustion the droplet size distribution, which depends on propellant formulation and combustion and on its own distributed combustion, is strongly polydisperse [11]. Moreover, it can dramatically change because of coalescence, that is the amalgamation of two droplets after a collision. But coalescence intensity depends on droplet velocities, which are conditioned by size. So droplet size and velocity are intimately intricate, which will be referred to as the "size/velocity coupling". The crucial importance of polydispersity and size/velocity coupling has been demonstrated in general cases in [17] and in solid rocket motor (SRM) cases in $[7,6]$. Accurate treatment of polydispersity, and of what controls it, is required since the size parameter directly drives the droplet-gas interactions and therefore instabilities. The Williams-Boltzmann equation allows to describe polydisperse, dilute and moderately dense liquid sprays by solving the evolution of the dispersed phase number density function (NDF) due to advection, drag force and heat transfer that couple the gaseous phase, and droplet-droplet interactions such as coalescence $[16,19,7]$. We focus here on moderately dense sprays, which have a significant enough mass fraction to strongly perturbate the gas but a small enough volume fraction to avoid particle volume effects. Moreover, coalescence occurs in many moderately dense sprays and is an issue in SRM.

A first strategy is to approximate the NDF by discrete numerical parcels of particles of various sizes through a Lagrangian Monte-Carlo approach [1], which is generally considered to be the most accurate for solving the Williams-Boltzmann equation. But it introduces diffusion when coupled to a Eulerian fluid and is difficult to parallelize. Collisions and coalescence can be treated in this context, usually with a stochastic approach $[16,19]$ but here again, accuracy and performance are difficult to reconcile. As an alternative the Eulerian Multi-Fluid model discretizes the NDF using a finite volume approach of the size variable but assumes size/velocity correlations to reduce the phase space dimension [18]. After integration on size intervals, the so-called "sections", the resulting conservation equations are similar to those of the pressureless gas dynamics (PGD), with source terms accounting for gas-particle and particle-particle interactions. The resolution of spray dynamics and coalescence coupled to the gaseous flow field can be performed with one size moment [19] or two size moments to approximate the size distribution in each section. The latter, introduced in [10] in order to treat evaporation, is well-suited in SRM cases as demonstrated in [7]. It is indeed a good compromise between high-order moment methods yielding accuracy but algebraic complexity -for instance on size-velocity coupling [29]- and modeling flexibility allowing to solve complex configurations. To solve the complex interactions of acoustic waves in a polydisperse spray without being limited by the smallest droplet dynamic time scales, a new splitting algorithm for which accuracy and stability were proven, was implemented for the Eulerian approach in the CEDRE code [9,8], a multi-physics platform on general unstructured meshes, for both research and industrial applications, in the fields of energetics and propulsion [26]. A low diffusion transport scheme for the dispersed phase has recently been implemented in CEDRE, that allows to capture moderately dense spray structures. But the impact of the entire strategy in SRM has never been assessed.

The long term purpose of our research is to reach a predictive Euler-Euler solver for the large eddy simulation (LES) of SRM in order to resolve the complex dynamics of polydisperse sprays, coalescence, combustion, and acoustic waves interaction, which are at the origin of the pressure oscillations. The objective of the present paper is to qualify the methods implemented in CEDRE in a realistic configuration of a P230 SRM featuring instabilities. We will focus on the interaction of hydrodynamic gaseous flow instabilities coupled to a polydisperse spray segregation dynamics and coalescence, where the resolution of the spray size spectrum is crucial. Such a study is conducted on an under-resolved mesh, where the main flow structures and the main numerical peculiarities are still featured, with a special attention

Email address: francois.doisneau@onera.fr (F. Doisneau). 
devoted to the fact that the conclusions to be drawn for this study are valid for a larger simulation, be it in the framework of DNS or LES. Besides, since a Lagrangian as well as the Two Size Moment Eulerian Multi-Fluid method have been implemented in the CEDRE code, we perform a comparison of the two approaches regarding cost, ease of use and accuracy. Let us insist on the fact that the proposed EulerEuler formalism and strategy provide for the first time a reliable resolution of all the physical phenomena involved, with algorithms that have the potential to be extended to realistic cases.

The paper is organized as follows : section 1 describes Eulerian and Lagrangian modeling of a coalescing spray from their common basis at the kinetic level. Section 2 describes recent improvements in Eulerian simulations to capture accurately the two-way coupling and the spray structures despite the high inhomogeneities that are vacuum and dense zones. Section 3 provides quantitative accuracy and cost comparisons between the Euler-Euler and Euler-Lagrange frameworks on a SRM without and with coalescence. Finally section 4 compares the advantages and drawbacks of both approaches for SRM simulation.

\section{Eulerian and Lagrangian modeling for coalescing sprays}

\subsection{A common kinetic basis : the Williams equation}

Let us define the number density function (NDF) $f$ of the spray, where $f(t, \mathbf{x}, \mathbf{u}, T, S) \mathrm{d} \mathbf{x} \mathrm{d} S \mathrm{~d} T \mathrm{~d} \mathbf{u} \mathrm{de}-$ notes the average number of droplets (in a statistical sense) at time $t$, in a volume of size dx around a space location $\mathbf{x}$, with a velocity $\mathbf{u}$, internal temperature $T$, and size $S$ in a $\operatorname{dud} T \mathrm{~d} S$-neighborhood of $(\mathbf{u}, T, S)$. The evolution of the NDF is described by the classical Williams-Boltzmann equation. Considering local momentum and heat transfer with the gas due to drag forces and conducto-convective fluxes and considering collisions between droplets but no evaporation nor break-up, it reads :

$$
\partial_{t} f+\mathbf{u} \cdot \partial_{\mathbf{x}} f+\partial_{\mathbf{u}} \cdot(\mathbf{F} f)+\partial_{T}\left(\frac{\mathrm{H}}{c_{p}(T)} f\right)=\Gamma
$$

where $\mathbf{F}$ is the drag force per unit mass, $H$ is the heat transfer per unit mass and $\Gamma$ is the collision source term. These terms require models which should take into account physical phenomena at the droplet scale, which specific heat capacity is $c_{p}(T)$. They are typically modeled by Stokes' laws with eventually Schiller-Nauman or Ranz-Marshall corrections, yielding characteristic dynamic and thermal times $\tau^{\mathbf{u}}(S)$ and $\tau^{T}(S)$ strongly depending on droplet size. Regarding collisions, we assume [19,7] :

[C1] We only take binary collisions into account.

[C2] The mean collision time is very small compared to the intercollision time.

[C3] Mass, momentum and droplet enthalpy are preserved during collisions.

[C4] Every collision leads to coalescence.

The collision operator resulting from these hypotheses is developed as $\Gamma=Q^{+}-Q^{-}$, respectively corresponding to creation and destruction of droplets due to coalescence. Considering two precursor droplets of volumes $v^{\star}$ and $v^{\diamond}$ colliding to form a new droplet of volume $v$, the operators read [16]:

$$
\begin{aligned}
Q^{+} & =\frac{1}{2} \int_{T^{\star}} \iint_{\mathbf{u}^{\star}, v^{\star} \in[0, v]} f\left(t, \mathbf{x}, \mathbf{u}^{\diamond}, T^{\diamond}, v^{\diamond}\right) f\left(t, \mathbf{x}, \mathbf{u}^{\star}, T^{\star}, v^{\star}\right) \mathfrak{B}\left(\left|\mathbf{u}^{\diamond}-\mathbf{u}^{\star}\right|, v^{\diamond}, v^{\star}\right) J \mathrm{~d} v^{\star} \mathrm{d} \mathbf{u}^{\star} \mathrm{d} T^{\star} \\
Q^{-} & =\int_{T^{\star}} \iint_{\mathbf{u}^{\star}, v^{\star}} f(t, \mathbf{x}, \mathbf{u}, T, v) f\left(t, \mathbf{x}, \mathbf{u}^{\star}, T^{\star}, v^{\star}\right) \mathfrak{B}\left(\left|\mathbf{u}-\mathbf{u}^{\star}\right|, v, v^{\star}\right) \mathrm{d} v^{\star} \mathrm{d} \mathbf{u}^{\star} \mathrm{d} T^{\star}
\end{aligned}
$$

where $v^{\diamond}=v-v^{\star}, v^{\diamond} \mathbf{u}^{\diamond}=v \mathbf{u}-v^{\star} \mathbf{u}^{\star}, v^{\diamond} h\left(T^{\diamond}\right)=v h(T)-v^{\star} h\left(T^{\star}\right)$ are pre-collisional parameters, $J=\left(v / v^{\diamond}\right)^{n_{d}+1} c_{p} / c_{p}^{\diamond}$ is the Jacobian of the mapping $(v, T, \mathbf{u}) \rightarrow\left(v^{\diamond}, T^{\diamond}, \mathbf{u}^{\diamond}\right)$ with $n_{d}$ the dimension of the velocity phase space, $c_{p}=c_{p}(T)$ and $c_{p}^{\diamond}=c_{p}\left(T^{\diamond}\right)$ and $\mathfrak{B}$ is the collision/coalescence probability kernel : 


$$
\mathfrak{B}\left(\left|\mathbf{u}-\mathbf{u}^{\star}\right|, v, v^{\star}\right)=\left|\mathbf{u}-\mathbf{u}^{\star}\right| \beta\left(v, v^{\star}\right) \mathfrak{E}\left(\left|\mathbf{u}-\mathbf{u}^{\star}\right|, v, v^{\star}\right) .
$$

In this kernel, $\beta\left(v, v^{\star}\right)=\pi\left(r+r^{\star}\right)^{2}$ is the impact parameter or geometric cross-section and $\mathfrak{E}$ is the collision efficiency. This efficiency accounts for the effects of the surrounding gas flow. Experimental results in SRM conditions suggest to use such models [5] to avoid significant over-estimations on coalescence intensity. In the following, we use a Langmuir-Blodgett law to get a reasonable coalescence intensity and to prove that these models are compatible with Eulerian approaches. The coalescence terms (2) are referred to as ballistic since their collision probability kernel is based on the resolved particle velocities. They are typical of DNS. In a LES context, sub-grid scale models (SGS) models should include coalescence terms linked to SGS velocity differences, often described as a statistical dispersion.

\subsection{Lagrangian approach}

A Lagrangian or stochastic particle method is implemented in the CEDRE code and consists in approximating the NDF with a finite number $I$ of parcels $i$ representing several droplets with the same position $\mathbf{x}_{p i}$, size $S_{p i}$, velocity $\mathbf{u}_{p i}$ and temperature $T_{p i}$, as follows :

$$
f(t, \mathbf{x}, \mathbf{u}, T, S)=\sum_{i \in[1, I]} w_{p i} \delta\left(\mathbf{x}-\mathbf{x}_{p i}(t)\right) \delta\left(S-S_{p i}(t)\right) \delta\left(\mathbf{u}-\mathbf{u}_{p i}(t)\right) \delta\left(T-T_{p i}(t)\right)
$$

where $w_{p i}$ is a weight. Practically in CEDRE, Eq. (1) is not solved but splitted in three steps [24] that are transport in the physical and phase space, collision and fragmentation, which is not considered here. In the transport step, the r.h.s. of (1) decouples for each parcel $i$ in the following system of ODE :

$$
\partial_{t} \mathbf{x}_{p i}=\mathbf{u}_{p i} ; \quad \partial_{t} S_{p i}=0 ; \quad \partial_{t} \mathbf{u}_{p i}=\mathbf{F}_{p i} ; \quad \partial_{t} T_{p i}=H_{p i} .
$$

which has to be solved with a method that respects the coupling to the gaseous phase. An unconditionally stable first order scheme is chosen to do so. In the collision step, pairs of particles are randomly chosen among each cell and their state is modified according to an algorithm described in $[16,19]$.

On the one hand, particle trajectories can be accurately integrated because they depend only on time so numerical diffusion is very limited. Moreover the method allows any type of particle trajectory crossings (PTC), especially those occurring on symmetry axes, close to a wall or at the rim of vortices. On the other hand, more particles are needed when degrees of freedom such as polydispersity or unsteadiness are solved which is costly in terms of CPU and memory. Moreover numerical diffusion is still introduced by the gas two-way coupling and the collision algorithm since source terms have to be evaluated on the Eulerian mesh. Finally, in a high performance computing context, possibilities of vectorization/parallelization or implicitation are limited. The particle load balance is particularly difficult to achieve [15].

\subsection{Two size Moment Eulerian Multi-Fluid approach}

To solve Eq. (1) with an affordable Eulerian approach, we need to reduce the size of the phase space so we only consider the moments of $f$ of order zero and one in the velocity and temperature variables. To close the system the following assumptions are introduced for a given droplet size $S$, at $(t, \mathbf{x})$ :

[H1] There is only one velocity $\overline{\mathbf{u}}(t, \mathbf{x}, S)$ and no dispersion.

[H2] There is only one temperature $\bar{T}(t, \mathbf{x}, S)$ and no dispersion.

Hypothesis [H1] is known as the monokinetic hypothesis [18]. It is equivalent to reducing the velocity distribution support to a one dimensional sub-manifold parameterized by droplet size. Hypothesis [H2] is

similarly introduced so that temperatures are locally correlated for droplets of a given size, which is, as soon as the monokinetic hypothesis holds, correct when $\tau^{T}(S)$ is close or smaller than $\tau^{\mathbf{u}}(S)$. We then 
choose a discretization $0=S_{0}<S_{1}<\cdots<S_{N_{\mathrm{sec}}}=\infty$ for the droplet size phase space and we average the conservation laws for number, velocity and enthalpy over each fixed size interval $\left[S_{k-1}, S_{k}[\right.$, called section. The set of droplets in one section can be seen as a "fluid" for which conservation equations are written, the sections exchanging mass, momentum and enthalpy. We also assume :

[H3] In each section, the velocity does not depend on the size of the droplets.

[H4] In each section, the temperature does not depend on the size of the droplets.

[H5] In each section, the form of $n$ as a function of $S$ is presumed.

We choose for assumption [H3] the notation $\overline{\mathbf{u}}(t, \mathbf{x}, S)=\overline{\mathbf{u}}_{k}(t, \mathbf{x})$ to designate the constant velocity distribution in section $k$. Hypothesis [H4] is similarly introduced here and is equivalent to presuming $\bar{h}(t, \mathbf{x}, S)=\bar{h}_{k}(t, \mathbf{x})$ as an enthalpy distribution in section $k$, and the corresponding effective temperature $\bar{T}_{k}(t, \mathbf{x})$ which allows to define the sectional specific heat capacity $\bar{c}_{k}=c_{p}\left(\bar{T}_{k}\right)$. Finally, all these hypotheses are equivalent to presume the following form of NDF :

$$
f(t, \mathbf{x}, \mathbf{u}, T, S)=\sum_{k=1}^{N_{\mathrm{sec}}} \kappa_{k}(t, \mathbf{x}, S) \delta\left(\mathbf{u}-\overline{\mathbf{u}}_{k}(t, \mathbf{x})\right) \delta\left(T-\bar{T}_{k}(t, \mathbf{x})\right) .
$$

The Two Size Moment Multi-Fluid model, described in [10] and extended to coalescence in [7], is based on a two-coefficient exponential reconstruction : $\kappa_{k}(t, \mathbf{x}, S)=a_{k}(t, \mathbf{x}) \exp \left(-b_{k}(t, \mathbf{x}) S\right)$ for $S \in\left[S_{k-1}, S_{k}[\right.$, where $\left(a_{k}(t, \mathbf{x}), b_{k}(t, \mathbf{x})\right)_{k}$ are chosen to preserve the following set of moments $\left(n_{k}(t, \mathbf{x}), m_{k}(t, \mathbf{x})\right)_{k}$. Finally the two-phase flow is described by a system including gaseous Navier-Stokes equations coupled to the conservation equations for the chosen moments. It is written here with Euler equations for simplicity :

$$
\left\{\begin{array}{l}
\partial_{t} \rho_{g}+\partial_{\mathbf{x}} \cdot\left(\rho_{g} \mathbf{u}_{g}\right)=0 \\
\partial_{t}\left(\rho_{g} \mathbf{u}_{g}\right)+\partial_{\mathbf{x}} \cdot\left(\rho_{g} \mathbf{u}_{g} \otimes \mathbf{u}_{g}+p \mathbf{I}\right)=-\sum_{k=1}^{N_{\mathrm{sec}}} m_{k} \overline{\mathbf{F}}_{k} \\
\partial_{t}\left(\rho_{g} e_{g}\right)+\partial_{\mathbf{x}} \cdot\left(\rho_{g} e_{g} \mathbf{u}_{g}\right)+p \partial_{\mathbf{x}} \mathbf{u}_{g}=-\sum_{k=1}^{N_{\mathrm{sec}}} m_{k} \overline{\mathrm{H}}_{k}-\sum_{k}^{N_{\mathrm{sec}}} m_{k} \overline{\mathbf{F}}_{k}\left(\mathbf{u}_{g}-\overline{\mathbf{u}}_{k}\right) \\
\partial_{t} n_{k}+\partial_{\mathbf{x}} \cdot\left(n_{k} \overline{\mathbf{u}}_{k}\right)=\mathrm{C}_{k}^{n+}-\mathrm{C}_{k}^{n-} \\
\partial_{t} m_{k}+\partial_{\mathbf{x}} \cdot\left(m_{k} \overline{\mathbf{u}}_{k}\right)=\mathrm{C}_{k}^{m+}-\mathrm{C}_{k}^{m-} \\
\partial_{t}\left(m_{k} \overline{\mathbf{u}}_{k}\right)+\partial_{\mathbf{x}} \cdot\left(m_{k} \overline{\mathbf{u}}_{k} \otimes \overline{\mathbf{u}}_{k}\right)=m_{k} \overline{\mathbf{F}}_{k}+\mathbf{C}_{k}^{m u+}-\mathbf{C}_{k}^{m u-} \\
\partial_{t}\left(m_{k} \bar{h}_{k}\right)+\partial_{\mathbf{x}} \cdot\left(m_{k} \bar{h}_{k} \overline{\mathbf{u}}_{k}\right)=m_{k} \overline{\mathrm{H}}_{k}+\mathrm{C}_{k}^{m h+}-\mathrm{C}_{k}^{m h-}
\end{array}\right\} \quad k=1, N_{\mathrm{sec}}
$$

where $\overline{\mathbf{F}}_{k}$ and $\overline{\mathrm{H}}_{k}$ are averaged on the section from their kinetic expressions. The coalescence source terms come from the expressions (2) that have been integrated on velocity thanks to the monokinetic hypotheses and on the two colliding partner section size intervals thanks to the Multi-Fluid reconstruction hypotheses. They couple the sections together but they are local in the physical space, analogous to chemical terms for instance. The size-integration method is crucial and has been carefully developed in the CEDRE code regarding computational performance : it is based on an adaptive quadrature technique that can handle smooth as well as steep and even singular size distributions [7]. 


\section{Numerical methods for Euler-Euler simulations}

\subsection{Achieving two-way coupling in moderately dense sprays}

A numerical method based on operator splitting is suggested in $[9,8]$ to ensure a robust and accurate two-way coupling for moderately dense sprays. It is referred to as Acoustic-Convective Splitting (ACS) since it has been designed to treat efficiently the fast scales of acoustics in a two-phase context. Its benefits on cost and accuracy have been quantified on two-phase acoustics in [9,8]. In complex flows however, twoway coupling unsteadiness has a similar sensitivity to polydispersity so the method is a priori well adapted for SRM cases. The method is briefly described here, as well as the treatment of coalescence in this context. The ACS is a two stage splitting based on the following timescale hierarchy : liquid convection generally yields the slowest scales while the fastest scales are gas transport scales, which include acoustic waves. The relaxation scales, that are drag and heat transfers between the liquid and the gas, are to be linked to the acoustic scales in order to account for two-phase acoustics. System (5) is considered as composed of transport operators $\mathscr{T}_{g}$ and $\left(\mathscr{T}_{k}\right)_{k}$ for the fluids, a relaxation operator $\mathscr{R}$ and a coalescence operator $\mathscr{C}$. These operators apply on $\mathbf{U}(t)=\left[\rho_{g}, \rho_{g} \mathbf{u}_{g}, \rho_{g} h_{g},\left(n_{k}, m_{k}, m_{k} \overline{\mathbf{u}}_{k}, m_{k} \bar{h}_{k}\right)_{k=1, N_{\mathrm{sec}}}\right]^{t}(t) \in \mathbb{R}^{4 N_{\mathrm{sec}}+3}$. The transport operators read :

$$
\mathscr{T}_{g}\left\{\begin{array} { l } 
{ \partial _ { t } \rho _ { g } + \partial _ { \mathbf { x } } \cdot ( \rho _ { g } \mathbf { u } _ { g } ) = 0 } \\
{ \partial _ { t } ( \rho _ { g } \mathbf { u } _ { g } ) + \partial _ { \mathbf { x } } \cdot ( \rho _ { g } \mathbf { u } _ { g } \otimes \mathbf { u } _ { g } + p \mathbf { I } ) = 0 } \\
{ \partial _ { t } ( \rho _ { g } e _ { g } ) + \partial _ { \mathbf { x } } \cdot ( \rho _ { g } e _ { g } \mathbf { u } _ { g } ) + p \partial _ { \mathbf { x } } \mathbf { u } _ { g } = 0 }
\end{array} \quad \mathscr { T } _ { k } \left\{\begin{array}{l}
\partial_{t} n_{k}+\partial_{\mathbf{x}} \cdot\left(n_{k} \overline{\mathbf{u}}_{k}\right)=0 \\
\partial_{t} m_{k}+\partial_{\mathbf{x}} \cdot\left(m_{k} \overline{\mathbf{u}}_{k}\right)=0 \\
\partial_{t}\left(m_{k} \overline{\mathbf{u}}_{k}\right)+\partial_{\mathbf{x}} \cdot\left(m_{k} \overline{\mathbf{u}}_{k} \otimes \overline{\mathbf{u}}_{k}\right)=0 \\
\partial_{t}\left(m_{k} \bar{h}_{k}\right)+\partial_{\mathbf{x}} \cdot\left(m_{k} \bar{h}_{k} \overline{\mathbf{u}}_{k}\right)=0
\end{array}\right.\right.
$$

The local operators that are relaxation and coalescence ensure the coupling between all the "fluids" and all the sections respectively :

$$
\mathscr{R}\left\{\begin{array}{l}
\partial_{t} \rho_{g}=0 \\
\partial_{t}\left(\rho_{g} \mathbf{u}_{g}\right)=-\sum_{k=1}^{N_{\mathrm{sec}}} m_{k} \overline{\mathbf{F}}_{k} \\
\partial_{t}\left(\rho_{g} e_{g}\right)=-\sum_{k=1}^{N_{\mathrm{sec}}} m_{k} \overline{\mathrm{H}}_{k}-\sum_{k}^{N_{\mathrm{sec}}} m_{k} \overline{\mathbf{F}}_{k}\left(\mathbf{u}_{g}-\overline{\mathbf{u}}_{k}\right) \\
\partial_{t} n_{k}=0 \\
\partial_{t} m_{k}=0 \\
\partial_{t}\left(m_{k} \overline{\mathbf{u}}_{k}\right)=m_{k} \overline{\mathbf{F}}_{k} \\
\partial_{t}\left(m_{k} \bar{h}_{k}\right)=m_{k} \overline{\mathrm{H}}_{k}
\end{array}\right\} \quad \begin{aligned}
& \mathscr{C}=1, N_{\mathrm{sec}} \\
& \partial_{t} n_{k}=\mathrm{C}_{k}^{n+}-\mathrm{C}_{k}^{n-} \\
& \partial_{t}\left(m_{k} \overline{\mathbf{u}}_{k}\right)=\mathbf{C}_{k}^{m u+}-\mathrm{C}_{k}^{m-} \\
& \partial_{t}\left(m_{k} \bar{h}_{k}\right)=\mathrm{C}_{k}^{m h+}-\mathrm{C}_{k}^{m h-}
\end{aligned}
$$

We can rewrite system $(5)$ as an operator equation $\mathbf{U}^{\text {exact }}\left(t+\Delta t_{\mathrm{c}}\right)=\left[\mathscr{C}+\mathscr{R}+\mathscr{T}_{g}+\sum_{k=1}^{N_{\mathrm{sec}}} \mathscr{T}_{k}\right]\left(\Delta t_{\mathrm{c}}\right) \mathbf{U}(t)$ between $t$ and $t+\Delta t_{\mathrm{c}}$. We now introduce the structure of the first stage of ACS, performed here according to a Strang splitting :

$$
\mathbf{U}^{\text {Strang }}\left(t+\Delta t_{\mathrm{c}}\right)=\left[\mathscr{C}+\mathscr{R}+\mathscr{T}_{g}\right]\left(\frac{\Delta t_{\mathrm{c}}}{2}\right)\left[\sum_{k=1}^{N_{\mathrm{sec}}} \mathscr{T}_{k}\right]\left(\Delta t_{\mathrm{c}}\right)\left[\mathscr{C}+\mathscr{R}+\mathscr{T}_{g}\right]\left(\frac{\Delta t_{\mathrm{c}}}{2}\right) \mathbf{U}(t) .
$$

The convective splitting time step is chosen to allow liquid convection to occur at its physical timescale which matches numerical criterion of the transport scheme $\Delta t_{\mathrm{c}} \sim \bar{\tau}_{k}^{c}$. We then suggest to consider the 
relaxation and the gas transport as a two-phase acoustics operator $\mathscr{A}$. Compared to the strategy presented in $[9,8]$, the coalescence operator has been included at the acoustic level since it can be stiff in dense zones and it modifies the droplet sizes, which condition the other time steps. Since $\mathscr{C}$ has a high numerical cost, it has been included through a first order Lie splitting to spare resources, while the acoustic operator is built on a Strang splitting. On a time step $\Delta t_{\mathrm{a}}$ respectful of acoustics, we finally have :

$$
\left.\left[\mathscr{C}+\mathscr{R}+\mathscr{T}_{g}\right]\left(\Delta t_{\mathrm{a}}\right) \approx \mathscr{C}\left(\Delta t_{\mathrm{a}}\right) \mathscr{A} \Delta t_{\mathrm{a}}\right) \quad \text { with } \quad \mathscr{A}\left(\Delta t_{\mathrm{a}}\right)=\mathscr{R}\left(\frac{\Delta t_{\mathrm{a}}}{2}\right) \mathscr{T}_{g}\left(\Delta t_{\mathrm{a}}\right) \mathscr{R}\left(\frac{\Delta t_{\mathrm{a}}}{2}\right) .
$$

\subsection{Capturing spray structures}

Since the spray follows the PGD in DNS or has a weak pressure in LES, strong gradients can appear, depending on the flow topology. For instance vortices yield, as soon as droplets are inertial enough, a remarkable segregation corresponding to strong density gradients. It is crucial to preserve these spray structures to remain accurate and to achieve predictive simulations. Therefore, a high quality solution is required, either with refined meshes or with an accurate method [14,21]. To spare degrees of freedom and cost, a high order transport scheme, yielding low diffusion, can be used. This is rarely achieved in EulerEuler simulations : for instance most high order numerical methods developed for Euler or Navier-Stokes equations are ill-suited because of the peculiarities of PGD that are vacuum zones and $\delta$-shocks [4]. A specific method has been developed for spray transport on generic unstructured meshes in CEDRE. It is based on a second order MUSCL reconstruction with unstructured gradient limitation [3] and with a Godunov-type flux scheme, but adapted to PGD. A first order method has been used for most simulations in this paper but the second order method is used and specifically discussed in section 4.2.

\subsection{Towards Large Eddy Simulation}

It is now acknowledged that, in many problems, LES provides more predictive solutions compared to RANS, whereas DNS is most of the time out of reach. For SRM studies, LES requires refined 3D meshes and a SGS modeling [27,23] that is specific to two-phase flows. Some models are available in the community, even if they cover only a partial range of Stokes numbers [22]. However, we claim that their implementation is straightforward once the PGD equations can be resolved with enough numerical efficiency, accuracy and robustness, which is the purpose of the present contribution. Remarkably a hybrid strategy between PGD and velocity-dispersed zones has already been suggested [2]. In this paper, we do not achieve predictive simulations regarding models and meshes but we perform DNS-like simulations : this to validate methods that are a prerequisite to any LES. The question of a robust and high order transport scheme is crucial for resolved turbulent scales to be effectively captured. The coupling method as introduced above aims at solving robustly the transfers between the two phases' resolved scales and is designed to be directly applicable in the framework of LES. And ballistic coalescence -based on the resolved particle velocity differences as above- remains significant even when SGS collisions appear.

\section{Numerical simulation of instabilities in the P230 SRM}

In this section, we give numerical results from the CEDRE code on a 2D configuration which reproduces the P230 motor that equips Ariane 5. This motor features obstacle vortex shedding (VSO) due to the thermal protection and parietal vortex shedding (VSP), which occurs in long segmented motors [20]. The chamber can host acoustic modes and flows through a supersonic nozzle. The propellant is aluminized 
so the flow contains liquid droplets of aluminum oxide from a zone that is close to the wall down to the nozzle. The droplet size distribution is often considered as bimodal after combustion [25] so we choose to model the spray injection with non-burning aluminum oxide droplets of size $d_{1}$ and $d_{2}$.

\subsection{Numerical configuration}

We consider a 2D axisymmetric mesh of the P230 at a specific moment of the grain combustion which is shown in Fig. 1. It is an unstructured 45, 500 generic cell mesh and the computation is paralellized on 32 domains. We use a coarse mesh, compared to typical SRM meshes with several million points, only to assess the feasibility of complex simulations with an Euler-Euler approach. For this, we now consider either the possibility of coalescence or not. The simulation starts from a steady state of the single phase gaseous flow field. When the particles are injected, the pressure builds up to a new value of average pressure. The simulated time is long enough to perform a harmonic study of the instabilities. Gas and particles are injected at a fixed surface flow rate from the two walls representing the two propellant segments. The injected liquid flow rate is fairly the same for the two segments. The loading is high since the liquid mass fraction reads $30 \%$ at the injection and can reach much more in the accumulation zones so a two-way coupling approach is required. The small droplets (diameter $d_{1}$ ) represent $90 \%$ of the injected mass and the big droplets (diameter $d_{2}$ ) the remaining $10 \%$. The number concentration is around $10^{15}$ at the wall so coalescence is to be considered. Because of the high density of alumina, the liquid volume fraction is much smaller than one so the liquid volume occupation effect can be neglected, the spray is moderately dense and system (5) is relevant.

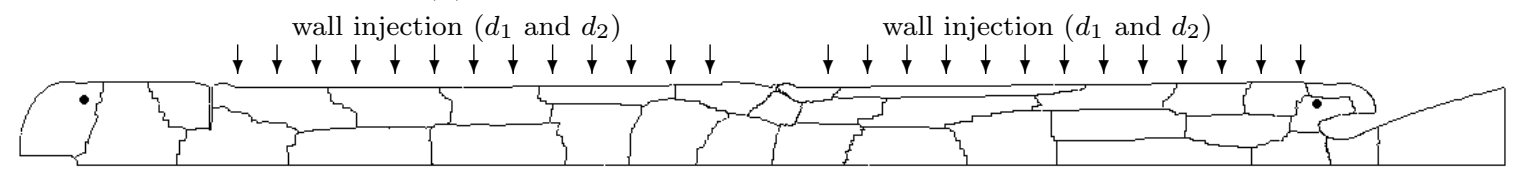

Figure 1. 2D axisymmetric geometry and domains of the P230 SRM (· sensor location).

The case is numerically costly because of the extreme stiffness yielded by the various space and time scales. Indeed the motor is more than $25 \mathrm{~m}$ long while the smallest hydrodynamic scales are a few centimeters. Its main instability mode is low frequency around $20 \mathrm{~Hz}$ while the small particle response time is close to the microsecond. The injection and nozzle output velocities, respectively a few $\mathrm{m} / \mathrm{s}$ and Mach 3, introduce time scales with a ratio of about 3,000 . The case is also costly because of the polydisperse coalescing liquid phase. First, it features a strongly bimodal disperse phase : because droplet characteristic times depend on the square of their diameters, this introduces two very different time scales, with a ratio of $10^{4}$. Second, the flow is injected at a very low velocity, encounters hydrodynamic and acoustic instabilities and finally crosses a supersonic nozzle with a high load ratio. We recapitulate all the transport characteristic times in Table 3.1. Since coalescence is a quadratic operator that is intrinsically costly, treating the transport stiffness by solving the smallest scales is definitely out of reach while reducing the cost with an implicit integration for instance would deteriorate accuracy on the unsteady phenomena. Thanks to the ACS splitting approach, the main time step can be chosen above the small particle response time : this allows to spare an order of magnitude on computational cost without compromising accuracy on the physics of small droplets while most classical coupling methods require a time step below the smallest particle scale for stability reasons $[9,8]$. The splitting strategy moreover decouples the coalescence intrinsic stiffness from the other time scales, allowing to treat it by a separate integration method if needed.

We now detail the numerical configuration for the size variable in each framework. In the Eulerian Two Size Moment method, the section discretization conditions the simulation's accuracy. For the inert case two sections are required to solve perfectly the bimodal distribution, no matter what bounds they have, thanks to the Two Size Moment method which can accurately approach the dynamics of any Dirac size 


\begin{tabular}{lr} 
Table 1. Timescale hierarchy $(\mu \mathrm{s})$, exhibiting stiffness. \\
\hline$d_{1}$ droplet Stokes time & 1 \\
Acoustic CFL time & 10 \\
Convective CFL time (nozzle) & 30 \\
$d_{2}$ droplet Stokes time & 10,000 \\
First acoustic mode & 50,000 \\
Eddy revolution time & 50,000 \\
Convective CFL time (injection) & 90,000 \\
Typical computation time & $1,000,000$ \\
\hline
\end{tabular}

distribution. And the two kinds of droplets will not change size during their transport. For the coalescing simulation, the monokinetic hypotheses prevents coalescence from occuring among droplets of the same section. This is relevant if the flow structure prevents PTC for droplets with fairly the same sizes. But the coalescence phenomenon does happen between different sections, which particles have different dynamics. Since the injected droplets are strongly bimodal and the flow yields particle acceleration, coalescence will occur between the two sections. The phase space is then discretized as follows. The first section is defined like in the inert case since no growth can occur. The second section now ranges from $d_{2}$ to $d_{3}=1.3 d_{2}$ to account for the big droplet size increase. A third section is also created to host even bigger droplets that can be created above $d_{3}$. A discretization as coarse as 3 sections has proven to be sufficient to capture basic coalescence size/dynamics coupling with the Two Size Moment Multi-Fluid method [7]. In the Lagrangian computations, the parametrization is the same as in [12]. Numerical particles of the two initial sizes are injected at each wall cell with a period of $4.10^{-4} \mathrm{~s}$. The number of particles per computational domain is however limited to 300,000 . Since we use 32 domains, this allows an average of 200 particles per cell which is said to be enough for the coalescence algorithm to converge [12].

\subsection{Flow topology}

Unsteadiness is visible on the gaseous vorticity field of the inert case (Fig. 2), where one retrieves a strong VSO and VSP along the wall, here tuned on the $1 \mathrm{~L}$ chamber mode. Both hydrodynamic instabilities are strongly coupled to the dispersed phase. The structure is the same with coalescence.

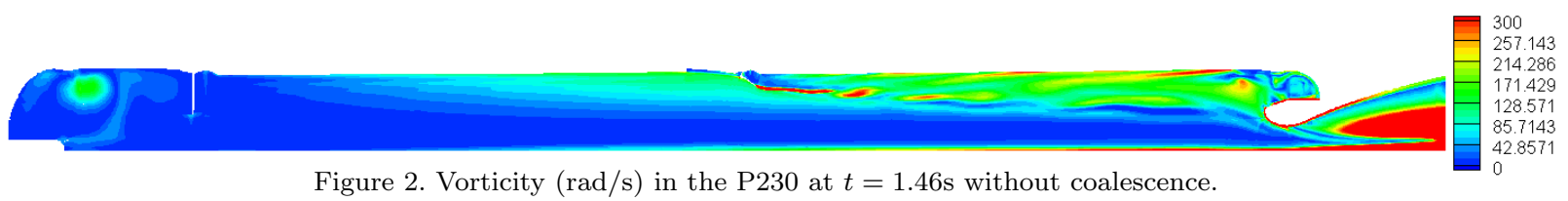

In the inert case we give Eulerian volume fractions in Fig. 3 and Lagrangian particle repartition in Fig. 4. The big droplets are segregated out of the vortices, while the small droplets fill most of the chamber. For the same reasons, small and big droplets flow differently throughout the nozzle. This dependence on size motivates a polydisperse approach. In the coalescing case and for the Eulerian simulation, the instantaneous volume fractions per section are in Fig. 5. This time, mass is transferred towards the third section, the big droplet mass being completely hosted in this section at the nozzle entrance. The Eulerian third section $d_{30}$ and the Lagrangian overall $d_{10}$ are presented as an illustration in Fig. 7. In both cases, the most intense coalescence zones are the edges of the vortices, the impact zone of the nozzle wall and the nozzle itself. Moreover coalescence can become so intense that droplet average sizes strongly vary through the domain. The droplet growth can however not be precisely compared since we have different average diameters on different size intervals. Finally Euler-Euler and Euler-Lagrange simulations give comparable droplet repartition and size. However quantitative comparison is made difficult by the radically different structure of output data. 


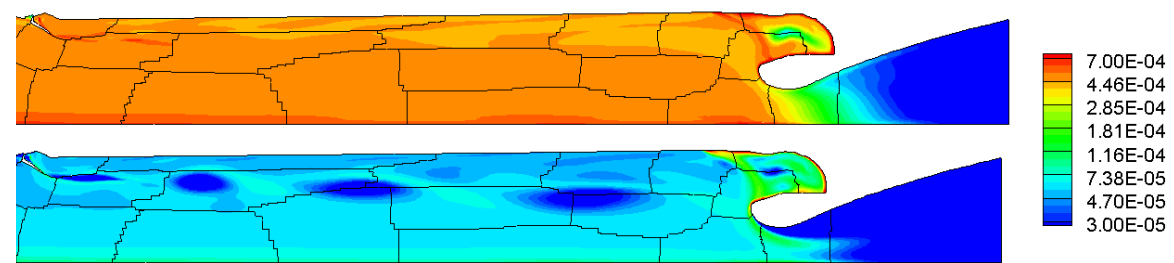

Figure 3. Volume fraction per section (Top : $d_{1}$; Bottom $: d_{2}$ ) at $t=1.09$ s without coalescence.

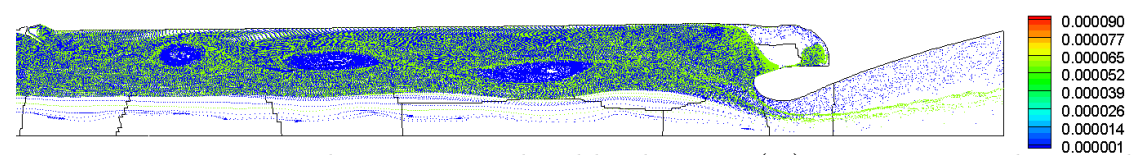

Figure 4. Lagrangian parcel repartition colored by diameter $(\mathrm{m})$ at $t=1.09 \mathrm{~s}$ without coalescence.

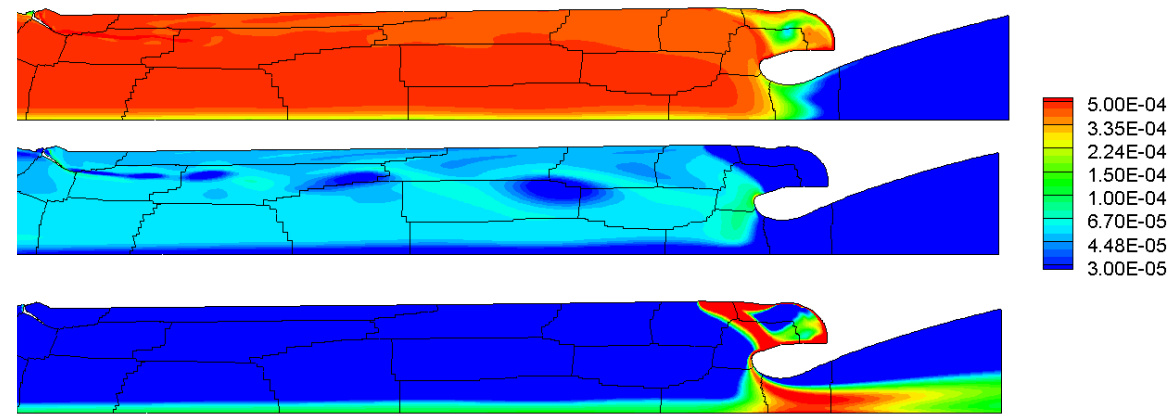

Figure 5. Volume fraction per section (Top : $d_{1}$; Middle $: d_{2}$ to $d_{3}$; Bottom : above $d_{3}$ ) at $t=0.81 \mathrm{~s}$ with coalescence.

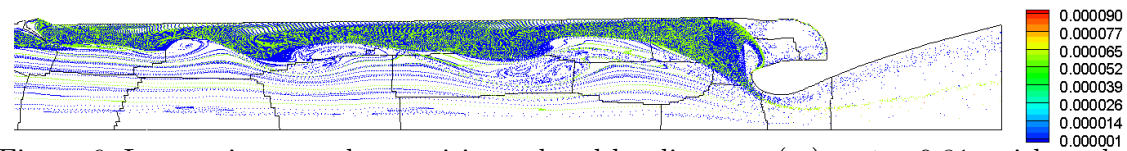

Figure 6. Lagrangian parcel repartition colored by diameter $(\mathrm{m})$ at $t=0.81 \mathrm{~s}$ with ${ }^{0.000000}$ escence.

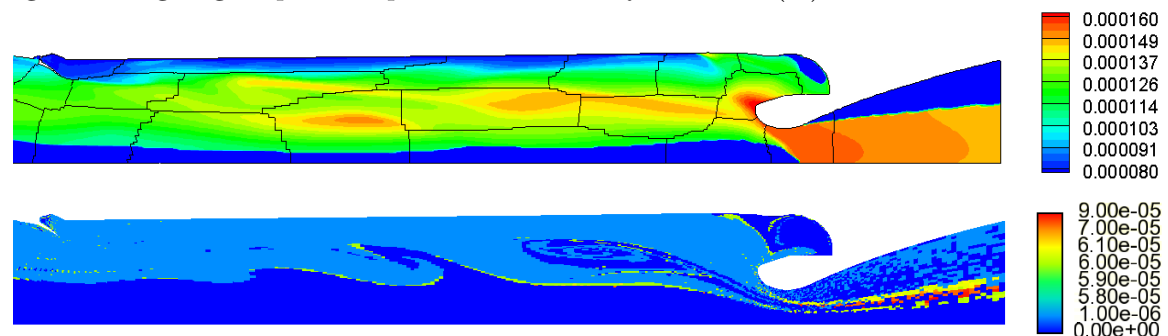

Figure 7. Droplet sizes with coalescence at $t=0.46 \mathrm{~s}$ - Top : Third section $d_{30}$ diameter $(\mathrm{m})$ for the Eulerian simulation; Bottom : Overall $d_{10}$ diameter $(\mathrm{m})$ for the Lagrangian simulation.

\subsection{Harmonic study}

The pressure oscillation depends strongly on the flow so we can use it to quantify the effect of coalescence and the differences between the two approaches. The time evolution of the front and rear pressure signals is now described : when the particle injection starts, the gaseous flow encounters an additional inertia so the chamber pressure drops whatever the evolution of droplet sizes. But when the droplets reach the nozzle, the flow slows down so that the pressure builds up. It reaches its initial level in the non-coalescing case but stabilizes about 2 bar lower for coalescing particles. We now focus on this permanent regime 
where the average pressure does not vary anymore and only the instability remains. The main values of the pressure signal at permanent regime are given in Tab. 2. Both Eulerian and Lagrangian approaches well agree on average pressure in the inert case and they predict a lower mean pressure at permanent state when coalescence has occurred. This pressure drop can be related to the particle growth. Small particles tend to be in equilibrium with the gas and to increase the chamber pressure while, for the same loading, larger particles tend to stress the deviation from equilibrium and have reduced effects on the pressure. In the asymptotic limit, i.e. for very large particles, the two-phase pressure is similar to the single-phase one. Having said that, a pressure drop of two bars seems to be quite important and may be the sign that coalescence is over-estimated. But regarding oscillation levels, they strongly disagree in the inert case, though they well agree in the coalescing case. The difference is such that the ability to be predictive is still at stake. Further studies are required to understand the differences on instability levels between Eulerian and Lagrangian simulations and to complete the cross validation.

Table 2. Non-dimensionned pressure data (Lagrangian values from ONERA simulations [12]).

\begin{tabular}{|c|c|c|c|c|}
\hline & \multicolumn{2}{|c|}{ Without coalescence } & \multicolumn{2}{|c|}{ With coalescence } \\
\hline & Lagrangian & Eulerian & Lagrangian & Eulerian \\
\hline Frequency & $1.00 \pm 1 \%$ & $1.02 \pm 4 \%$ & $0.99 \pm 2 \%$ & $1.10 \pm 4 \%$ \\
\hline Front sensor Average & 1.00 & 0.99 & 0.98 & 0.95 \\
\hline pressure & 1.0 & 4.7 & 1.8 & 1.6 \\
\hline
\end{tabular}

\section{Eulerian VS Lagrangian computations}

\subsection{Ergonomy of both approaches}

The ease to set and exploit a computation is assessed here. Lagrangian and Eulerian approaches have common points but they differ in their treatment of the size variable and on the form of the output data. Regarding size parametrization, an equivalent diameter approach is unsuitable in SRM as shown in [6] so it is compulsory to account for polydispersity. In this case of strongly bimodal distribution though, the size distribution is easy to model with two classes or two sections. Yet coalescence induces a size shift which has to be captured. The Multi-Fluid approach requires section bounds, which are determined from an a priori estimation of the droplet size evolution in the flow. This choice is generally difficult but any coarse but reasonable discretization is often satisfactory to get the dynamic trend as shown in [7]. The Lagrangian approach only requires enough particles to converge on the size distribution. Here again, this is difficult to determine a priori and it can only be evaluated thanks to numerical noise. Regarding coalescence computation now, Eulerian approaches compute deterministic integrals so they do not require particular care. On the contrary, Lagrangian collision algorithms are stochastic so they require sufficient particles per cell to converge. Finally most collision efficiency models can be used in both cases, as shown in [7] for the Eulerian method. Regarding post-processing, we highlight that Eulerian fields are convenient and mostly used so that Lagrangian output data require averaging operations.

\subsection{Numerical diffusion assessment}

Nonetheless is the flow better solved when the liquid structures are captured, but coalescence, which strongly depends on concentration, absolutely requires spatial accuracy. A transport scheme that is second order in space is currently developed in CEDRE for the Eulerian approach of the liquid phase. Since it has not been fully validated, it has not been used for the previous results but we have performed additional 
computations to make a qualitative comparison: the diffusion level improvement for the Eulerian approach compared to the Lagrangian approach is visible in Fig. 8, which shows a very good agreement on the particle structuration by the vortices for the high order Eulerian scheme. We insist on the fact that the finer the structures and the steeper the gradients are resolved, the more intense the coalescence and retrocoupling source terms are. The overall robustness of the two-phase numerical method is therefore challenged when higher accuracy is achieved for moderately dense sprays.
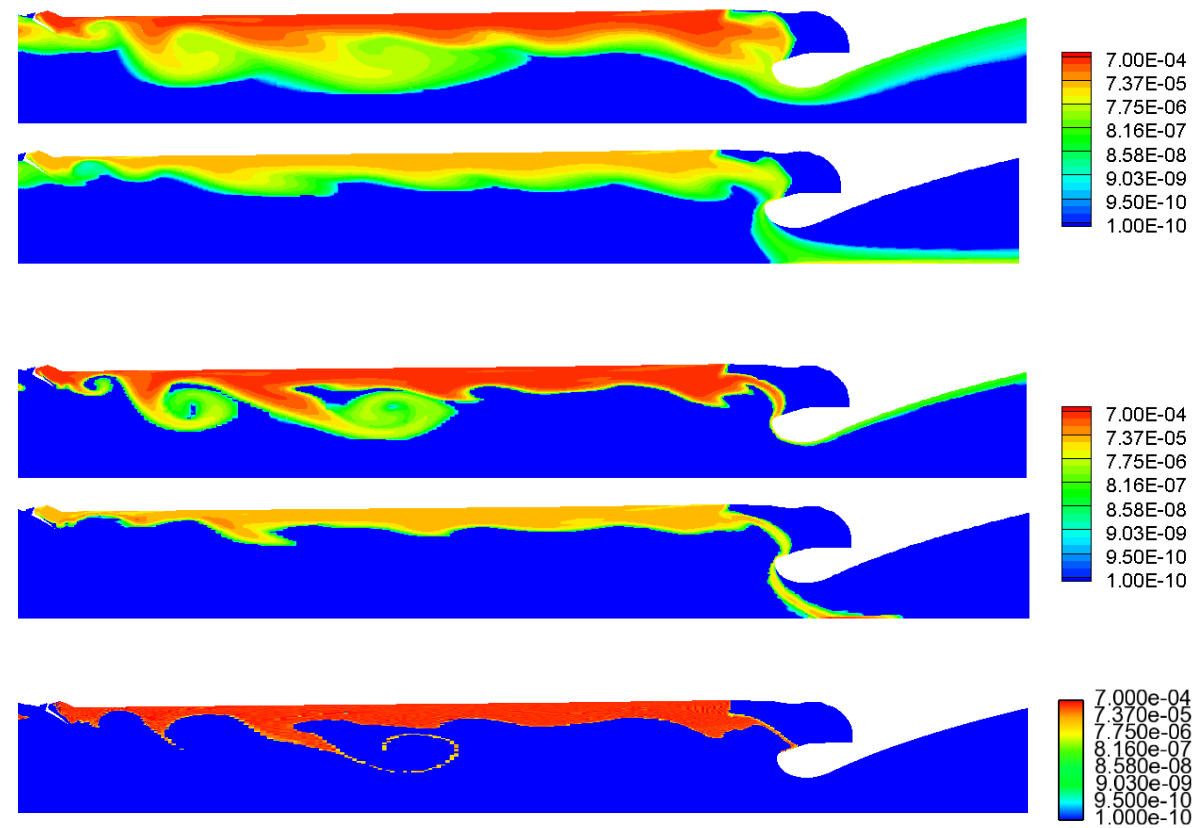

Figure 8. Volume fractions at $t=0.278 \mathrm{~s}$ without coalescence - Top : $d_{1}$ and $d_{2}$ with first order transport scheme; Middle : $d_{1}$ and $d_{2}$ with second order transport scheme; Bottom : both sizes with Lagrangian.

\subsection{Particle Trajectory Crossing treatment with Eulerian methods}

PTC is here considered for particles of the same size. It occurs in high strain zones, where the flow accelerates or curves faster than the particles can react. Critical Stokes numbers can be defined, depending on the configuration, above which PTC occurs. This has been tackled for Taylor Green vortices and Homogeneous Isotropic Turbulence $[4,14,17,28]$. The Eulerian Multi-Fluid approach fails to treat PTC because of the monokinetic hypothesis [H1] [4]. In the P230 chamber, PTC does not occur very much on the axis and the unresolved PTC effect is moderate at the edge of the vortices so the Eulerian approach is satisfactory. In the nozzle however an unphysical $\delta$-shock appears on the axis because of momentum averaging of the two symmetric particle jets. Moreover $\delta$-shocks densify as the mesh refines or as the scheme's diffusion decreases, which is well illustrated in Fig. 8 and is a major issue of both numerical convergence and physical meaning. Specific impulse loss can therefore be considered as mis-estimated. The monokinetic hypothesis generates the same kind of limitations at the wall than for PTC : the disperse phase can only slip along the wall with no normal velocity component because of momentum averaging. So the PTC failure of Eulerian mono-kinetic approaches is a major drawback for certain SRM applications at the wall and in the nozzle. It can only be treated by implementing a polykinetic approach, either with multiple velocities $[17,30]$ or with pressure-like terms that account for an average effect of PTC $[27,28]$, depending on the range of Stokes numbers. 


\subsection{Computational cost and parallelizability}

Finally, let us consider the cost of the two approaches as implemented in CEDRE on a complex SRM case. They are given in Table 3 and appear to be comparable. The major difference, highlighted here, is the natural scalability of the Eulerian method compared to the difficulty to increase the number of CPUs for Lagrangian computations as said previously. This is a major advantage of Euler-Euler approaches for two-phase flows. The cost of such simulations becomes indeed prohibitive on 3D meshes [13]. Moreover, convergence on particle repartition and two-way coupling is naturally achieved with Eulerian approaches while no criterion on particle number or integration time step is known for Lagrangian approaches.

\begin{tabular}{lll}
\multicolumn{2}{c}{ Table 3. Computational cost per time step (32 Nehalem CPU). } \\
\hline Eulerian & Lagrangian \\
\hline Inert & $84 \mathrm{~ms}$ & $124 \mathrm{~ms}$ \\
Coalescence & $144 \mathrm{~ms}$ & $236 \mathrm{~ms}$ \\
\hline
\end{tabular}

\section{Conclusion}

This paper brings three salient facts to light. First, considering coalescence in aluminized SRM simulations is incontrovertible because the droplet sizes impact on the liquid repartition, on instability levels, on specific impulse loss, and probably on slag weight. Second, the Euler-Euler approach is valid when compared to Lagrangian computations even though it has modeling weaknesses such as PTC treatment, as long as PTC occurs moderately. Third, the Euler-Euler approach is accessible and recommendable for two-way coupled simulations, for parallelization purpose. Finally, further validations are required to completely match with Lagrangian references in such complex configurations. Then the model and method can bear a transposition to LES in order to achieve accessible and predictive simulations.

\section{Acknowledgements}

This research was done thanks to a Ph. D. grant from DGA, Ministry of Defence (M. S. Amiet, Technical Monitor) and a France-Stanford grant from the French Ministry of Foreign Affairs (M. Massot, Technical Monitor). The authors also thank J.-L. Estivalezes (ONERA) for preliminary Lagrangian computations and SME for the P230 mesh.

\section{References}

[1] G. A. Bird. Molecular gas dynamics and the direct simulation of gas flows. Oxford Science Publications, $42,1994$.

[2] M. Boileau, C. Chalons, F. Laurent, S. de Chaisemartin, and M. Massot. Robust numerical schemes for Eulerian spray DNS and LES in two-phase turbulent flows. Center for Turbulence Research, pages 359-370, 2010.

[3] B. Courbet, C. Benoit, V. Couaillier, F. Haider, M.C. Le Pape, and S. Péron. Space discretization methods. Aerospace Lab, 2:1-14, 2011.

[4] S. de Chaisemartin. Polydisperse evaporating spray turbulent dispersion : Eulerian model and numerical simulation. PhD thesis, Ecole Centrale Paris, 2009. Available at http://tel.archives-ouvertes.fr/tel-00443982/en/.

[5] F. X. D'Herbigny and P. Villedieu. Etude expérimentale et numérique pour la validation d'un modèle de coalescence. Technical Report RF1/05166 DMAE, ONERA, 2001.

[6] F. Doisneau, F. Laurent, J. Dupays, and M. Massot. Two-way coupled simulation of acoustic waves in polydispersed coalescing two-phase flows : application to Solid Rocket Motor instabilities. In $4^{\text {th }}$ EUCASS, pages 1-10, 2011. 
[7] F. Doisneau, F. Laurent, A. Murrone, J. Dupays, and M. Massot. Eulerian Multi-Fluid models for the simulation of dynamics and coalescence of particles in solid propellant combustion. J. of Comp. Physics (accepted for publication), pages 1-37, 2012. Available at http://hal.archives-ouvertes.fr/hal-00618806.

[8] F. Doisneau, A. Sibra, J. Dupays, A. Murrone, F. Laurent, and M. Massot. Numerical strategy for two-way coupling in unsteady polydisperse moderately dense sprays. submitted to J. Prop. Power, pages 1-31, 2012. Available on HAL.

[9] F. Doisneau, A. Sibra, F. Laurent, J. Dupays, and M. Massot. Numerical strategy for two-way coupling in unsteady polydisperse moderately dense sprays. In $47^{\text {th }}$ AIAA Joint Propulsion Conf., pages 1-20, 2011.

[10] G. Dufour and P. Villedieu. A second-order Multi-Fluid model for evaporating sprays. M2AN Math. Model. Numer. Anal., 39(5):931-963, 2005.

[11] J. Dupays. Two-phase unsteady flow in solid rocket motors. Aerospace Science and Technology, 6(5):413-422, 2002.

[12] J.-L. Estivalezes. Simulations lagrangiennes polydisperses avec collisions et coalescence sur une configuration Ariane V 2D à l'instant 107s. Technical Report RT 3/17717 DMAE, ONERA, 2010.

[13] J.-L. Estivalezes. Simulations lagrangiennes polydisperses avec et sans collision-coalescence sur une configuration Ariane V tridimensionnelle à l'instant 107s. Technical Report RT 10/17717 DMAE, ONERA, 2011.

[14] L. Fréret, O. Thomine, J. Reveillon, S. de Chaisemartin, F. Laurent, and M. Massot. On the role of preferential segregation in flame dynamics in polydisperse evaporating sprays. Proceedings of the CTR Summer Program 2010, pages 383-392, 2010. Available at http://www.stanford.edu/group/ctr/Summer/SP10/6_08_freret.pdf.

[15] M. Garcia. Développement et validation du formalisme Euler-Lagrange dans un solveur parallèle non-structuré pour la simulation aux grandes échelles. PhD thesis, Institut National Polytechnique de Toulouse, 2009.

[16] J. J. Hylkema and P. Villedieu. A random particle method to simulate coalescence phenomena in dense liquid sprays. In Proc. 16th Int. Conf. on Num. Meth. in Fluid Dyn., volume 515, pages 488-493, Arcachon, France, 1998.

[17] D. Kah, F. Laurent, L. Fréret, S. de Chaisemartin, R. Fox, J. Reveillon, and M. Massot. Eulerian quadrature-based moment models for dilute polydisperse evaporating sprays. Flow Turbulence and Combustion, 85(3-4):649-676, 2010.

[18] F. Laurent and M. Massot. Multi-fluid modeling of laminar poly-dispersed spray flames: origin, assumptions and comparison of the sectional and sampling methods. Comb. Theory and Modelling, 5:537-572, 2001.

[19] F. Laurent, M. Massot, and P. Villedieu. Eulerian Multi-Fluid modeling for the numerical simulation of coalescence in polydisperse dense liquid sprays. J. Comp. Phys., 194:505-543, 2004.

[20] N. Lupoglazoff and F. Vuillot. Parietal vortex shedding as a cause of intstability for long solid propellant motors. numerical simulations and comparisons with firing tests. AIAA Paper 96-0761, January 1996.

[21] L. Martinez. Simulation aux grandes échelles de l'injection de carburant liquide dans les moteurs à combustion interne. PhD thesis, Institut National Polytechnique de Toulouse, 2009.

[22] E. Masi. Étude théorique et numérique de la modélisation instationnaire des écoulements turbulents anisothermes gaz-particules par une approche Euler-Euler. PhD thesis, Institut National Polytechnique de Toulouse, 2010.

[23] M. Moreau, O. Simonin, and B. Bédat. Development of gas-particle Euler-Euler LES approach: A priori analysis of particle sub-grid models in Homogeneous Isotropic Turbulence. Flow, Turb. and Comb., 84(2):295-324, 2009.

[24] A. Murrone and P. Villedieu. Numerical modeling of dispersed two-phase flows. Aerospace Lab, 2:1-13, 2011.

[25] E. W. Price. Combustion of Metallized Propellants, pages 479-513. AIAA, 1984. Ed. K.E. Kuo and M. Summerfield.

[26] A. Refloch, B. Courbet, A. Murrone, P. Villedieu, C. Laurent, P. Gilbank, J. Troyes, L. Tessé, G. Chaineray, J.B. Dargaud, E. Quémerais, and F. Vuillot. CEDRE software. Aerospace Lab, 2:1-10, 2011.

[27] M. Simoes. Modélisation eulérienne de la phase dispersée dans les moteurs à propergol solide, avec prise en compte de la pression particulaire. PhD thesis, Institut National Polytechnique de Toulouse, 2006.

[28] A. Vié, F. Doisneau, F. Laurent, and M. Massot. On the anisotropic gaussian closure for the prediction of inertial-particle laden flows. Submitted to Comm. in Comp. Phys., pages 1-18, 2012.

[29] A. Vié, F. Laurent, and M. Massot. Size-velocity correlations in high order moment methods for polydisperse evaporating sprays : modelling and numerical issues. Submitted to J. of Comp. Physics (in revision), pages 1-36, 2012.

[30] C. Yuan and R.O. Fox. Conditional quadrature method of moments for kinetic equations. J. Comp. Physics, 230(22):8216-8246, 2011. 